\title{
Electromyographic analysis in upper limbs of Brazilian air force flight instructors submitted to maneuvers in a T-27 force simulator
}

\begin{abstract}
Aim: The specific professional activity of pilots is dependent of health status. Health hazards associated with acrobatic flight may have negative aspects, since pilots do not always receive precise preparation for certain situations. Together with a complex position and an increased gravitational load, the acrobatic movements place high demand on skeletal muscles and circulatory system. The aim of this study was to evaluate the mean and peak electromyographic amplitude of upper limbs in Brazilian Air Force flight instructors submitted to a reproduction of acrobatic maneuvers (Looping, Tonneau Barrel, Tonneau Slow, Returnemant) using the Force Simulator Prototype (SFA-EMB312/T27). This study is of fundamental importance to prevent shoulder injuries in Brazilian military pilots. The incidence of detachment and injury due to the high forces generated in the joystick is very high.
\end{abstract}

Methods: Twelve male flight instructors participated in the study. Surface electromyography was used to analyse ten muscles of the upper limbs during four movements (the front, the back, external rotation and internal rotation) on a Force Simulator Prototype (SFAEMB312/T27). The Root Mean Square (RMS) of the signal, expressed in microvolt's $(\mathrm{mV} ;$ mean $\pm \mathrm{SD})$, was calculated through the electromyographic amplitude of each selected muscle in all maneuvers.

Results: There were higher mean electromyographic activation (EMGa) for the Anterior Deltoid muscle $(162,280 \pm 60 ; \mathrm{mV})$ in the front movement; the Flexor Carpi Radialis muscle $(123,349 \pm 36 ; \mathrm{mV})$ in the back movement; the Brachial Biceps muscle $(314,587 \pm 73 ; \mathrm{mV})$ in the Internal Rotation movement; and the Posterior Deltoid muscle $(545,028 \pm 125,5 ; \mathrm{mV})$ in the External Rotation movement. The mean EMGa indicated greater total muscle activation $(132,592 \pm 157 ; \mathrm{mV})$ in the External Rotation movement. Results of peak EMGa showed that the highest values were presented for the Anterior Deltoid muscle $(334,234 \pm 109 ; \mathrm{mV})$ in the front movement; the Flexor Carpi Radialis muscle $(407,969 \pm 114 ; \mathrm{mV})$ in the back movement; the Brachial Biceps muscle $(440,263 \pm 120 ; \mathrm{mV})$ in the Internal Rotation movement; and the Posterior Deltoid muscle $(1277,229 \pm 333,5 ; \mathrm{mV})$ in the External Rotation movement.

Conclusion: Our data demonstrated that some maneuvers within acrobatic flights require a higher demand for specific muscles of the upper limbs. Further research is required to define the influence of physical training as well as volume, intensity and optimum loads to improve pilot performance and safety, and to prevent injury during acrobatic flights.
Volume 2 Issue 3 - 2018

\author{
Bezerra TAR, 1,2 Guindani G, ,', Campos \\ FAD, ${ }^{1,2}$ Romero R, ${ }^{2}$ Santiago PRP ${ }^{3}$ \\ 'Brazilian Air Force, Brazil \\ 2Postgraduate Program in Operational Human Performance, \\ Brazilian Air Force University, Brazil \\ ${ }^{3}$ School of Physical Education and Sport, University of São Paulo, \\ Brazil
}

Correspondence: Thiago Augusto Rochetti Bezerra, Postgraduate Program in Operational Human Performance, Brazilian Air Force University, Rio de Janeiro, Brazil, Email thiago_rochetti@globomail.com

Received: June 19,2017 | Published: May 092018

\section{Introduction}

The position adopted by pilots is complex. At the same time, pilots are required to maintain the body for the effects of increasing Gravitational load $(\mathrm{Gz})$ and use the senses of sight, touch and executing hand on the joystick, and feet on the pedals according to the actions needed, all this respecting the joint boundaries in a manner to avoid pain. These brutish and heavy joystick movements place high demand on the skeletal muscles of the pilots. ${ }^{1,2}$ There is a high rate of shoulder injury in Brazilian Air Force pilots flying on this type of T-27 aircraft (Figure 1). In this way, knowing the forces and muscles necessary for the physical training of injury prevention justifies the accomplishment of this study. Acrobatics and military flight maneuvers have been shown to expose the human organism to a great variation of pressure and muscular efforts..$^{2-4}$ Additionally, in military flight, it is common that the aircraft exceeds the constant gravity acceleration $\left(1 \mathrm{Gz}^{+}\right)$.At the Brazilian Air Force Academy (AFA), pilots are exposed to $\mathrm{Gz}^{+}$ load variations five times greater than gravity. Physical training it is suggested to contribute for injury prevention, considering the pilotspecific activities during flights.

Electromyography is a method of recording the electrical potential changes of a muscle, allowing access to the patterns of muscular electrical activity, and providing research on likely synergies, and the predominance of muscles in determined patterns of movement. ${ }^{5,6}$ Therefore, the objective of this study was to describe the electromyographic activity of upper-limbs muscles during (name of the maneuvers) in an aircraft T-27. The team of authors of this article works directly with the physical preparation in military pilots of the Brazilian Air Force. Thus, the interest in this study was based on the evidence of the high index of injuries in pilots and the lack of knowledge about the mechanical forces applied to the pilots during the accomplishment of maneuvers in this 


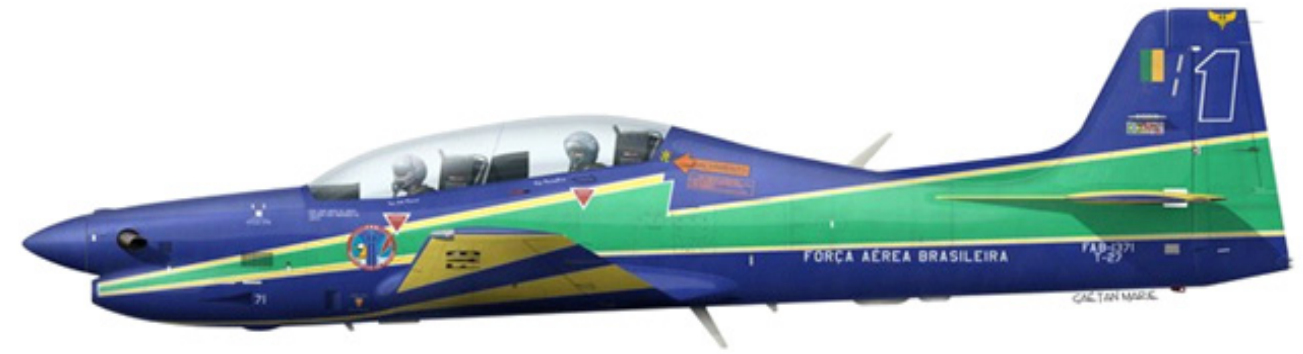

Figure I Aircraft T 27.

\section{Materials and methods}

\section{Participants}

Twelve male flight instructors of the Brazilian AFA (mean age: $28.8 \pm 2.5$ years; weight: $68.9 \pm 7.4 \mathrm{Kg}$; height: $1.73 \pm 0.04 \mathrm{~cm}$ ) participated voluntarily in this study. A signed express consent (Free and Informed Consent Term - TCLE) was obtained prior to starting participation, according to resolution 196/96 of CEP. Opinion Number: 1.015.756 Date of the Rapporteur: 04/14/2015, CAAE: 40667114.7.0000.5504.

\section{Inclusion/exclusion criteria}

Were considered suitable for this study were flight attendants who were approved in the Physical Fitness Assessment Test (TACF) regulated through the Systemic Standard of the Aeronautical Command (NSCA) 54-1 (2004), ${ }^{7}$ performed each semester in the AFA, and aviators who underwent an annual examination at the Aeronautical Medicine Center (CEMAL), the Aeronautical Health Organization (OSA), designated by the ANAC, through an agreement with the Aeronautical Command (COMAER), and obtained the Certificate of Physical Capacity ) In inspections. Were considered unable to participate in this study, the military that was disapproved, in any of the reports related to the Test of Assessment of the conditioning and medical evaluation, according to the Technical Instructions of the Aeronautical Health Inspections.

\section{Instrumentation}

A surface electromyography system (Trigno, Wireless EMG system, Delsysinc, Boston, MA, USA) was used to record muscle activity of the upper-limbs. The electric signal of the Prototype for the SFA-EMB312/T27 (University of Sao Paulo, Ribeirao Preto, SP, Brazil) was used for the skeletal muscles most active in performing maneuvers and acrobatics (Figure 2).

\section{Procedures}

Participants were informed on the procedures and objectives of the study. After participants have signed the informed consent, all procedures for data collection were conducted at the Laboratory of Biomechanics and Motor Control (LABIOCOM) of School of Physical Education and Sports of the University of São Paulo (EEFERP-USP). Prior to data collection, the skin was cleaned with isopropyl alcohol, upper limbs hair were scraped, and a light scraping of the skin was performed to remove dead cells. Thereafter, surface electrodes were placed on the belly of 10 muscles (flexor carpi radialis, extensor carpiulnaris, brachial biceps, brachial triceps, anterior deltoid, posterior deltoid, upper trapezius, pectoralis major, infraspinatus, and latissimus dorsi), following the Surface Electromyography for the Noninvasive Assessment of Muscles (SENIAM) recommendations (Figure 3A)(Figure 3B). Surface electromyography was used as it provides greater comfort for the volunteer and is easy to manage for the researcher. ${ }^{8}$

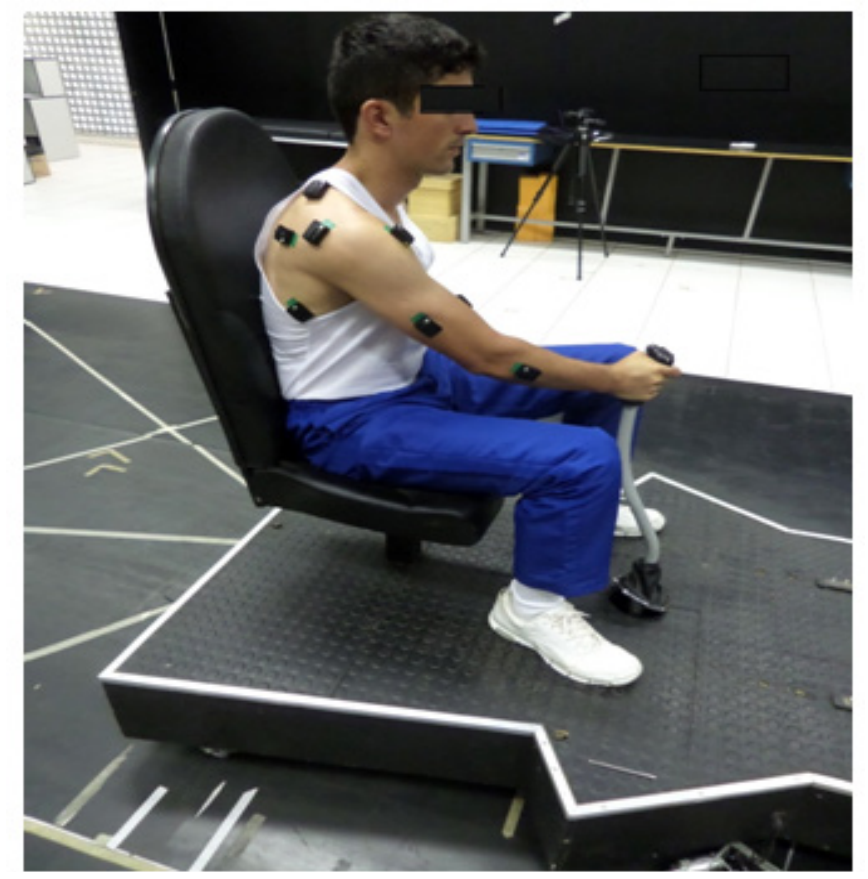

Figure 2 Lateral view of the force simulator.

The aim of this study was to evaluate the mean and peak electromyographic amplitude of upper limbs in Brazilian Air Force flight instructors submitted to a reproduction of acrobatic maneuvers (Looping, Tonneau Barrel, Tonneau Slow, Returnemant) using the Force Simulator Prototype (SFA-EMB312/T27). ${ }^{9}$ The maximum isometric contraction time was measured in the spot of this simulator, and was performed in the movements: 
a. At the front;

b. The back;

c. Rolling to the right;

d. Rolling to the left, respectively.

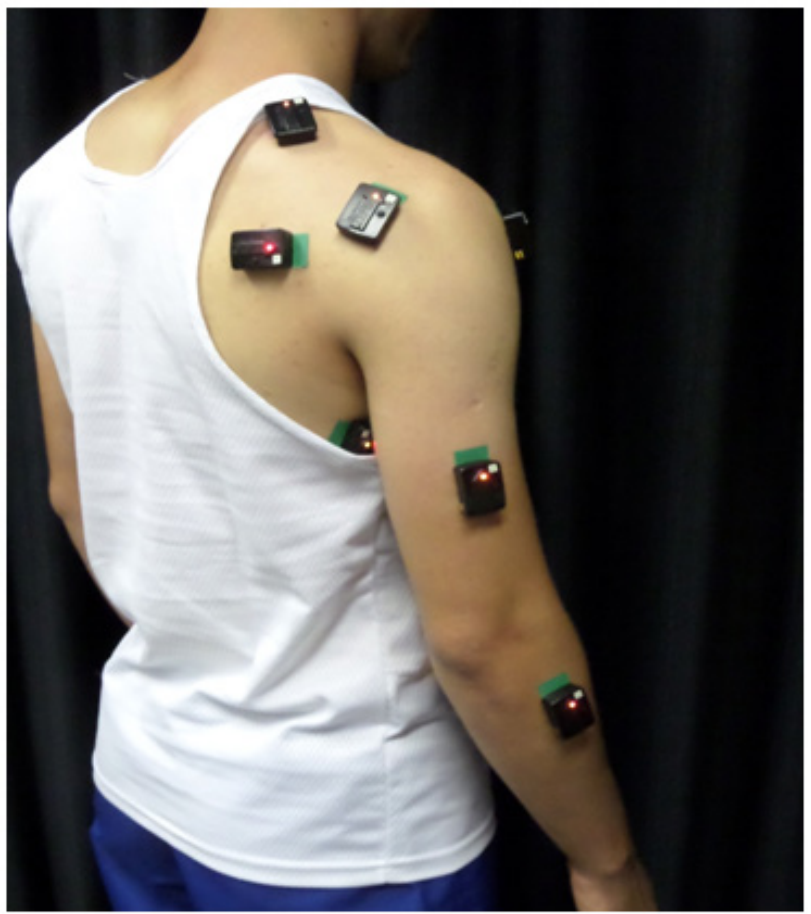

Figure 3A Placement of the electrodes following SENIAM recommendations.

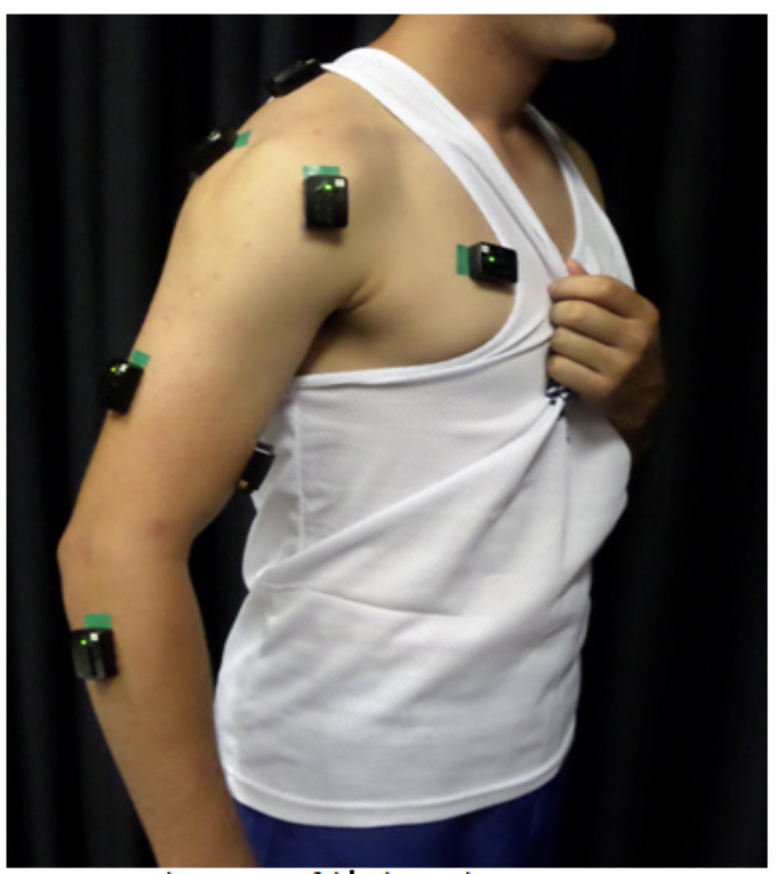

Figure 3B Placement of the electrodes.
The total time for each direction was 60 seconds. The load in Kgf in these movements was $30 \mathrm{kgf}$ and is equivalent to the maximum force applied to the spot during the execution of stunts on the T-27 aircraft subjected to a $5 \mathrm{Gz}+$ gravitational force. ${ }^{9}$ EMG recordings. The sampling frequency of the surface EMG signal was in the order of $2000 \mathrm{~Hz}$. The electrodes used were distance between the electrodes was $20 \mathrm{~mm}$ from center to center Figures 4-7 shows the execution of the analyzed movements. ${ }^{10}$

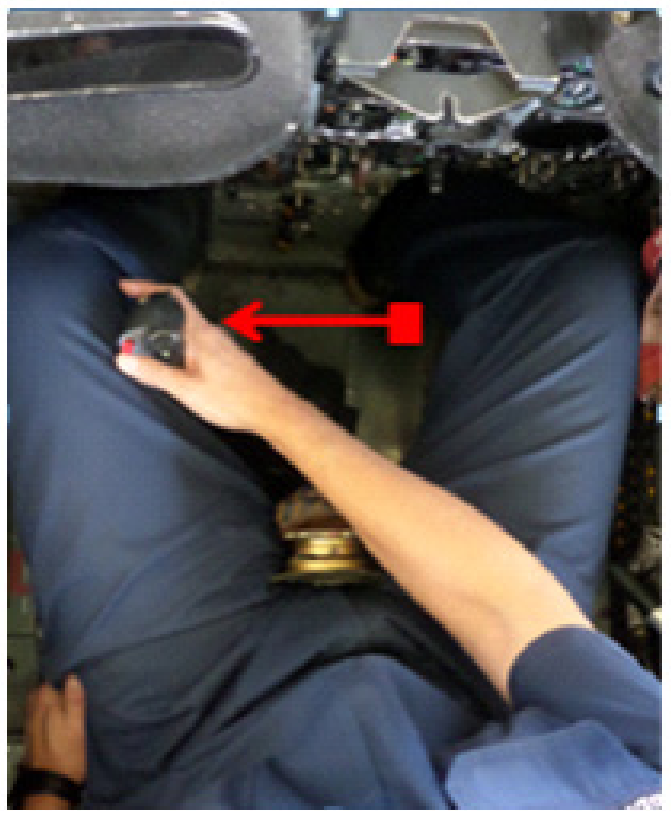

Figure 4 Rot left.

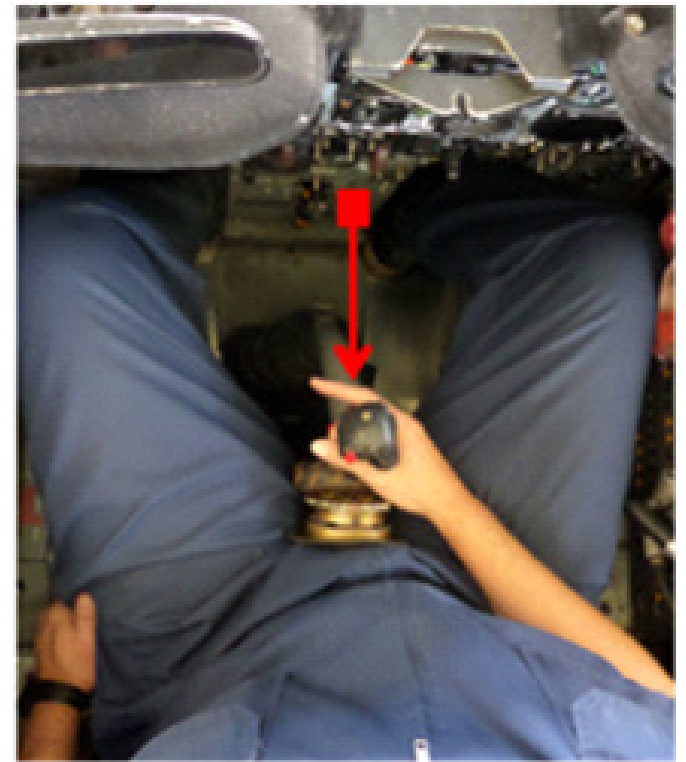

Figure $\mathbf{5}$ The back.

The root mean square (RMS) of the signals was analyzed through the electromyographic amplitude of each of the selected muscles in the front, the back, External Rotation, and Internal Rotation 
movements. The RMS values were smoothed in the program Delsystrigno wireless, exported to the format ".txt" and passed to the MatLab program, where a specific routine was used to remove the mean and peak values for each trial in each condition. The RMS data (Root Mean Square) found through the routine created in the MatLab program was normalized by the peak of the EMG signal of each volunteer. This methodological procedure was based on another study produced and already published by our team of researchers. ${ }^{14}$ The input signals were amplified up to 1000 times by the preamplifier in the cable and filtered within a frequency of $20 \mathrm{~Hz}$ to $800 \mathrm{~Hz}$ with active filters. The electromyography had a gain adjustment in the final stage and offset, which made it ideal for the system. The electromyographic electrodes used in this study captured the bipolar signal in the passive configuration, with an instrumentation amplifier INA 118 near the electrodes, amplifying the signal 1000 times near the signal source, thus avoiding degradation of the signal-to-noise ratio.

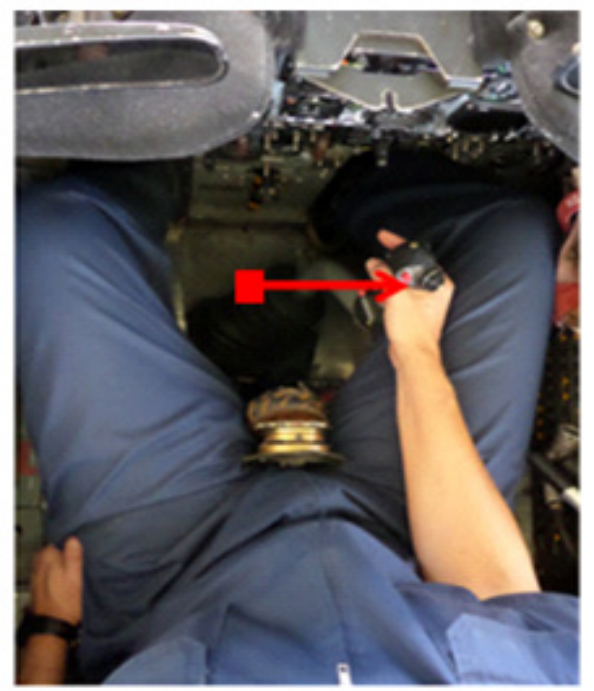

Figure 6 Rot right.

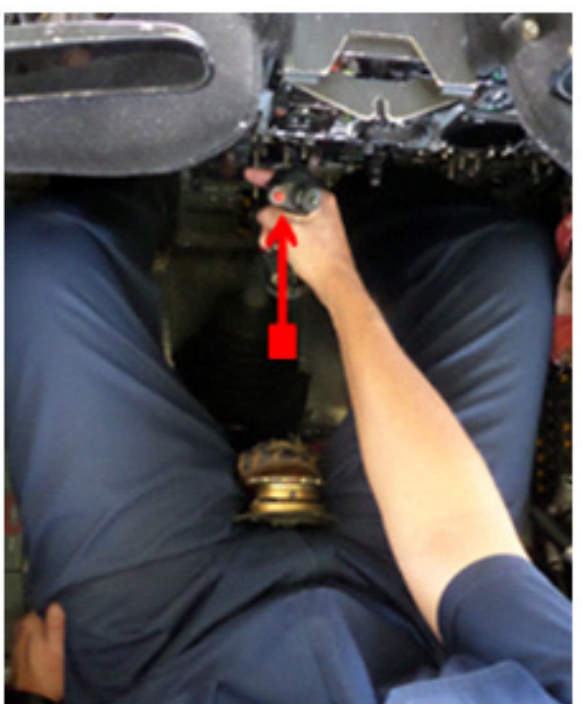

Figure 7 The front.

\section{Statistical analysis}

Descriptive statistics and outcome values were expressed by mean \pm standard deviation. All statistical analyses were performed using the Action Add-in from the Microsoft Excel (version, company, city). To analyze the data distribution, the Shapiro-Wilk normality test was applied.

\section{Results}

There was a higher mean electromyographic activation of the anterior deltoid muscle in the front movement $(162,280 \mathrm{mV})$; the flexor carpi radialis muscle in the back movement $(123,349 \mathrm{mV})$; the brachial biceps muscle in the Internal Rotation movement $(440,263 \mathrm{mV})$; and the posterior deltoid muscle in the external rotation movement $(545,028 \mathrm{mV})$. Through the analysis of the mean electromyographic activation, there was greater muscle activation in the external rotation movement $(1325,928 \mathrm{mV})$ followed by the internal rotation $(990,0680 \mathrm{mV})$, the back $(516,93 \mathrm{mV})$, and the front movements $(485,904 \mathrm{mV})$. Details on the mean and peak EMG activity of each muscle are available in Table $1 \&$ Table 2, respectively. The analysis of peak electromyographic activation showed that the highest values were presented for the Anterior Deltoid muscle $(334,234 \mathrm{mV})$ in the front movement; the Flexor Carpi Radial is muscle in the back movement $(407,969 \mathrm{mV})$, the Brachial Biceps muscle in the Internal Rotation movement $(314,587 \mathrm{mV})$, and the Posterior Deltoid Muscle in the External Rotation movement $(1277,229 \mathrm{mV})$. The analysis of the peak electromyographic activation showed greater muscle activation in the External Rotation movement $(3598,876 \mathrm{mV})$, followed by the Internal Rotation $(1598,964 \mathrm{mV})$, the back $(1371,476 \mathrm{mV})$, and the front movements $(993,856 \mathrm{mV})$ (Table 2).

Table I Upper-limbs EMG activity in microvolt's (mean \pm SD)

\begin{tabular}{|c|c|c|c|c|}
\hline & Front & Back & Int. Rotation & $\begin{array}{l}\text { Ext. } \\
\text { Rotation }\end{array}$ \\
\hline $\begin{array}{l}\text { Flexor Carpi } \\
\text { Radialis }\end{array}$ & 7,440 & 123,349 & 81,184 & $|82| 4 \mid$, \\
\hline $\begin{array}{l}\text { Extensor Carpi } \\
\text { Ulnaris }\end{array}$ & 37,092 & 25,752 & 41,724 & 39,354 \\
\hline Brachial Biceps & 9,659 & 54,043 & 314,587 & 20,926 \\
\hline Brachial Triceps & 52,088 & 18,723 & 40,771 & 62,312 \\
\hline Anterior Deltoid & 162,280 & 9,045 & 72,617 & 121,446 \\
\hline Posterior Deltoid & 105,173 & 110,030 & 13,536 & 545,028 \\
\hline Upper Trapezius & 8,709 & 59,506 & 9,955 & 125,662 \\
\hline Pectoralis Major & 6,005 & 7,517 & 225,504 & 9,693 \\
\hline Infra-spinal & 78,904 & 53,972 & 16,035 & 175,009 \\
\hline Latissimus Dorsi & 18,550 & 54,997 & 48,475 & 44,353 \\
\hline Mean & 48,590 & 51,693 & 86,439 & 132,592 \\
\hline Standard Deviation & 52,39 & 39,65 & 101,79 & 157,59 \\
\hline
\end{tabular}


Table 2 Analysis of the Peak Electromyographic amplitude in microvolt's (mean \pm SD)

\begin{tabular}{|c|c|c|c|c|}
\hline & Front & Back & $\begin{array}{l}\text { Int. } \\
\text { Rotation }\end{array}$ & $\begin{array}{l}\text { Ext. } \\
\text { Rotation }\end{array}$ \\
\hline Flexor Carpi Radialis & 21,693 & 407,969 & 268,368 & 572,583 \\
\hline $\begin{array}{l}\text { Extensor Carpi } \\
\text { Ulnaris }\end{array}$ & 55,785 & 55,431 & $|26,20|$ & 95,449 \\
\hline Brachial Biceps & 16,758 & 262,987 & 440,263 & 94,332 \\
\hline Brachial Triceps & 83,028 & 37,861 & 73,188 & 173,620 \\
\hline Anterior Deltoid & 334,234 & 27,925 & 238,549 & 432,402 \\
\hline Posterior Deltoid & 203,173 & 145,888 & 47,771 & $1,277,229$ \\
\hline Upper Trapezius & 21,403 & $|33,30|$ & 19,160 & 369,121 \\
\hline Pectoralis Major & 13,459 & 17,230 & 264,760 & 19,093 \\
\hline Infra-spinal & 206,431 & 156,022 & 39,222 & 470,104 \\
\hline Latissimus Dorsi & 37,886 & 126,859 & 207,154 & 94,938 \\
\hline Mean & 99,385 & 137,147 & 172,464 & 257,960 \\
\hline Standard Deviation & 110,38 & 121,37 & $|34,9|$ & 203,32 \\
\hline
\end{tabular}

\section{Discussion}

For a better understanding of muscle strength, the present study sought through the electromyographic analysis to find out which intensity the upper limb muscles are requested during the execution of simulated acrobatics.

The present study used EMG methods to suggest a specific training needed for pilots from the Brazilian Air Force Academy (AFA), in terms of muscular activation. There was higher mean electromyographic activation for the Anterior Deltoid muscle in the front movement $(162,280 \mathrm{mV})$; the Flexor Carpi Radial is muscle in the back movement; $(123,349 \mathrm{mV})$ the Brachial Biceps Muscle in the Internal Rotation movement $(440,263 \mathrm{mV})$, and the Posterior Deltoid Muscle in the External Rotation movement $(545,028 \mathrm{mV})$. Through the analysis of the mean electromyographic activation, there was greater muscle activation in the External Rotation movement $(1325,928 \mathrm{mV})$ followed by the Internal Rotation $(990,0680 \mathrm{mV})$, the back $(516,93 \mathrm{mV})$, and the front movements $(485,904 \mathrm{mV})$. The analysis of the peak electromyographic activation demonstrated that the highest values were presented for the Anterior Deltoid muscle $(334,234 \mathrm{mV})$ in the front movement; the Flexor Carpi Radial is muscle in the back movement $(407,969 \mathrm{mV})$, the Brachial Biceps Muscle in the Internal Rotation movement $(314,587 \mathrm{mV})$, and the posterior Deltoid Muscle in the External Rotation movement $(1277,229 \mathrm{mV})$.

The analysis of the peak electromyographic activation demonstrated greater muscle activation in the External Rotation movement $(3598,876 \mathrm{mV})$, followed by the Internal Rotation $(1598,964 \mathrm{mV})$, back $(1371,476 \mathrm{mV})$, and front movements $(993,856 \mathrm{mV})$. The overload is increased when the pilot is subjected to $\mathrm{aGz}+$ force during acrobatic maneuvers. ${ }^{9}$ This force is external and compresses the pilot in the seat, increasing the effort necessary during movement of the limbs. This overload can cause pain and injury. ${ }^{2-4}$ The acceleration force is proportional to the velocity and inversely proportional to the angle of the curve that the aircraft performs. As it is a compressive force that can reach a value of $5 \mathrm{Gz}+$ during acrobatic maneuvers, it causes an increase in body weight and body segments by up to 5 times (according to the value of $\mathrm{Gz}^{+}$), exposing pilots to severe joint and muscular overloads that may cause injuries. ${ }^{11}$ Adaptations are highly necessary, and the lack of specific physical training can reduce unequal flight conditions, causing postural alterations in pilots. ${ }^{13,14} \mathrm{As}$ the fleet of EMB 312 T-27-Tucano aircraft at the Brazilian Air Force (BAF) is very large with a high manufacturing cost and no forecast for replacement with newer models, there is a need for adequate adaptation of the pilots to this aircraft. In this way, the pilots can have favorable conditions to pilot, without greater physical overloads and with satisfactory conditions to eject in case of extreme necessity. ${ }^{12-14}$ Knowledge of the morphological characteristics of BAF pilots is of great value in the development of new aircraft designs and in adaptations of aircraft already manufactured or acquired.

\section{Acknowledgements}

None.

\section{Conflict of interest}

The authors declare that there is no conflict on interest.

\section{References}

1. Mierau A, Girgenrath M, Bock O. Isometric force production during changed-Gz episodes of parabolic flight. Eur J Appl Physiol. 2008;102(3):313-318.

2. Albery WB. Acceleration in other axes affects $+\mathrm{Gz}$ tolerance: dynamic centrifuge simulation of agile flight. Aviat Space Environ Med. 2004;75(1):1-6.

3. Whinnery T, Forster EM. The $+\mathrm{Gz}$-induced loss of consciousness curve. Extrem Physiol Med. 2013;2(1):19.

4. Tripp LD, Warm JS, Matthews G, et al. +Gz acceleration loss of consciousness: Time course of performance deficits with repeated experience. Hum Factors. 2006;48(1):109-120.

5. Paz GA, Maia MF, Lima VP, et al. Maximal exercise performance and electromyography responses after antagonist neuromuscular proprioceptive facilitation: a pilot study. $J$ Exerc Physiol. 2012;15(6):60-67.

6. Ramírez A, Garzón DA. Sensitivity analysis for the positioning of electrodes in surface electromiography (semg). Rev Fac Ingla Univ Antioquia. 2008;46:70-79.

7. Defense Ministry. Systemic Rule of Aeronautics Command 54-1: Physical Fitness Assessment Test. Brazil: Aeronautics Command; 2004.

8. Bellomo R, Zoccolella S, Lapenna F, et al. Electromyography as usefultest in the differential diagnosis of neuromuscular diseases only after clinical examination. Clinical Neurophysiology. 2013;124(11):e205.

9. Bezerra TAR, Shimano AC, Campos FAD. Analysis of the forces exerted in flight by Aviator Cadets of the Brazilian Air Force. Journal of Aeronautical Sciences. 2014;5(2):61-67.

10. Han WQ, Hu WD, Dong MQ, et al. Cerebral hemodynamics and brain functional activity during lower body negative pressure. Aviat Space Environ Med. 2009;80(8):698-702. 
11. Merletti R, Parker P. Electromyography: Physiology, Engineering and Noninvasive Applications. New Jersey: John Wiley \& Sons; 2004. 506 p.

12. Newsom BD, Goldenrath WL, Winter WR, et al. Tolerance of females to $+\mathrm{G}(\mathrm{z})$ centrifugation before and after bed rest. Aviat Space Environ Med. 1977;48:327-331.
13. Schlegel TT, Wood SJ, Brown TE, et al. Effect of $30-\mathrm{min}+3 \mathrm{Gz}$ centrifugation on vestibular and autonomic cardiovascular function. Aviat Space Environ Med. 2003;74(7):717-724.

14. Bezerra TARB, Shimano AC, Campos FAD. Analysis of the forces exerted in flight by Aviator cadets of the Brazilian Air Force. Journal of Aeronautics Sciences. 2014;5(2):61-67. 